\title{
In vivo efficacy of doripenem (DRPM) against Pseudomonas aeruginosa in murine chronic
}

\section{respiratory tract infection model}

\section{Nobuko Araki $^{\mathrm{a}}$, Katsunori Yanagihara ${ }^{\mathrm{a}, \mathrm{b}}$, Yoshitomo Morinaga ${ }^{\mathrm{a}, \mathrm{b}}$, Koichi Yamada ${ }^{\mathrm{a}, \mathrm{b}}$, Yasuaki}

Yamada $^{\mathrm{a}}$, Shigeru Kohno ${ }^{\mathrm{b}, \mathrm{c}}$ and Shimeru Kamihira ${ }^{\mathrm{a}}$

${ }^{\mathrm{a}}$ Department of Laboratory Medicine and ${ }^{\mathrm{b}}$ Second Department of Internal Medicine, Nagasaki

University Graduate School of Biomedical Sciences, ${ }^{\circ}$ Global COE Program, Nagasaki University,

Nagasaki, Japan

short title: In vivo efficacy of doripenem against $P$. aeruginosa

Address correspondence to:

Katsunori Yanagihara, MD, PhD

Department of Laboratory Medicine, Nagasaki University School of Medicine, 1-7-1 Sakamoto,

Nagasaki 852-8102, Japan.

Tel.:+8195819 7418; Fax: +81958197257

E-mail address: k-yanagi@nagasaki-u.ac.jp (K. Yanagihara). 
Araki et al., Page 2 of 14

\section{Abstract}

Doripenem is a carbapenem antibiotic with broad-spectrum coverage of gram-positive and gram-negative bacteria, including Pseudomonas aeruginosa, and is considered to be as effective as meropenem. The in vivo activity of doripenem was thus compared with that of meropenem in a chronic lower respiratory P. aeruginosa infection mouse model. The number of viable bacteria in the lungs after treatment with doripenem, meropenem, and saline mice was $2.01 \pm 0.69,2.03 \pm 0.48$ and $3.90 \pm 1.40 \log _{10} \mathrm{CFU} / \mathrm{lung}$, respectively. The number of viable bacteria in the lungs of mice treated with doripenem and meropenem was significantly lower than that in lungs of controls.

Histopathological examination of lung specimens from the control group revealed promotion of the inflammatory response in chronic bronchial infection. However, the groups treated with doripenem and meropenem showed weaker inflammatory responses. These results suggest that doripenem treatment is effective against chronic airway infection with P. aeruginosa.

Key words; doripenem, carbapenem, chronic respiratory infection, mouse model, lung,

\section{Pseudomonas aeruginosa}


Araki et al., Page 3 of 14

\section{Introduction}

Pseudomonas aeruginosa is often involved in chronic lower respiratory tract infectious

conditions, such as cystic fibrosis, diffuse panbronchiolitis, chronic obstructive pulmonary disease (COPD) and ventilator-associated pneumonia. After P. aeruginosa colonizes the lower respiratory tract, it is difficult to treat. Chronic respiratory infection causes unnecessary inflammation and lung tissue damage in humans, leading to decreased lung function. Subsequently, P. aeruginosa may cause acute exacerbation of chronic respiratory infection, and this constitutes the main cause of mortality among patients affected by this condition [1]. Such patients require treatment with antibiotics at every acute exacerbation event.

Doripenem (DRPM) is a carbapenem antibiotic with broad spectrum activity against gram-positive and gram-negative bacteria, including P. aeruginosa, and it also has potent in vitro activity against many multidrug-resistant hospital pathogens [1-3]. Against some pathogens, the activity of DRPM is slightly higher than that of meropenem(MEPM) and imipenem(IPM) [1,2,4-6], and the chemical structure of DRPM is very similar to that of MEPM. DRPM and MEPM, unlike IPM, both have a 1-beta-methyl side chain that provides stability against human renal dehydropeptidase-I (DHP-I). In DRPM, however, the dimethylcarbamoyl side chain of MEPM is replaced with a sulfamoylaminomethyl group. The $\mathrm{MIC}_{90} \mathrm{~s}$ of DRPM, MEPM and IPM against the 
clinical isolates of P. aeruginosa from hospitals located in the Americas and Europe are 0.5, 1 and 2 $\mu \mathrm{g} / \mathrm{ml}$, respectively [1].

In this study, we investigated the efficacy of DRPM, as compared to MEPM, in a chronic lower respiratory tract $P$. aeruginosa infection model.

\section{Materials and methods}

\subsection{Antimicrobial agents}

DRPM was kindly provided by Shionogi \& Co., Ltd. (Osaka, Japan). MEPM was provided by Dainippon Sumitomo Pharma Co., Ltd. (Osaka, Japan). Both agents were dissolved in saline.

\subsection{Laboratory animals}

Male, ddY, specific pathogen-free mice (age, 5 - 6 weeks; body weight, 30 - $35 \mathrm{~g}$ ) were purchased from Shizuoka Agricultural Cooperative Association Laboratory Animals (Shizuoka, Japan). All animals were housed in a pathogen-free environment and received sterile food and water in the Laboratory Animal Center for Biomedical Science at Nagasaki University. The experimental 
Araki et al., Page 5 of 14

protocol was approved by the Ethics Review Committee for Animal Experimentation at our institution.

\subsection{Bacterial strains}

P. aeruginosa S10 strain, which was isolated from sputum of patiants with chronic respiratory infection at Nagasaki University Hospital, was used in this study. S10 is a mucoid strain, and we have reported that S10 is suitable strain to make a murine model of chronic respiratory infection in the previous study [7],[8]. Bacteria were stored at $-80^{\circ} \mathrm{C}$ in Microbank ${ }^{\mathrm{TM}}$ (Pro-Lab Diagnostics Inc., Toronto, Canada) until use.

\subsection{Antibiotic susceptibility testing}

The MICs of the agents were determined by the broth dilution method with Mueller-Hinton broth (Becton Dickinson and Company, Franklin Lakes, NJ). Microtiter plates containing $5.0 \times 10^{4}$ $\mathrm{CFU} /$ well were incubated with agents at $37^{\circ} \mathrm{C}$ for $24 \mathrm{~h}$ and the lowest concentration of agent that prevented visible growth was considered to be the MIC.

\subsection{Experimental model of chronic respiratory infection}


Disposable sterile plastic cut-down intravenous catheters with a $3 \mathrm{Fr}$. (1 mm) outer diameter (Atom Co., Tokyo, Japan) were used for intubation. The tubes were $5.0 \mathrm{~mm}$ in length, with a few slits made at the proximal end to prevent blockage by oral secretions. To prepare inoculum, $P$. aeruginosa was cultured on a Muller-Hinton II agar plate for $24 \mathrm{~h}$, and bacteria were then suspended in saline, harvested by centrifugation $\left(3,000 \times g, 4^{\circ} \mathrm{C}, 10 \mathrm{~min}\right)$, resuspended in sterile saline and adjusted to $2 \times 10^{9} \mathrm{CFU} / \mathrm{ml}$, as estimated by turbidimetry. The intubation tube was then immersed in the bacterial saline suspension for 3 days at $37^{\circ} \mathrm{C}$. The bacterial count on these tubes 3 days after incubation and just prior to intubation was $6.0 \pm 0.3\left(\log _{10} \mathrm{CFU} / \mathrm{ml}\right.$, mean $\left.\pm \mathrm{SD}, \mathrm{n}=5\right)$.

The method used for inducing infection has been described in detail previously [7],[8]. Briefly, the intubation tube harbouring bacteria was attached to the blunted tip of the needle of an intravenous catheter (Angiocath; Beckton Dickinson, Vascular Access Sandy, UT). The needle-tube was inserted through the oral cavity, and was then advanced through the vocal cords. When the tip of the tube was in the trachea, the needle/catheter was pulled out and the outer sheath was pushed gently to place the pre-coated tube into the main bronchus.

\subsection{Treatment protocol}

Lower airway infection was induced in mice with P. aeruginosa, as described above. DRPM or MEPM was injected intraperitoneally into the mice twice a day $(100 \mathrm{mg} / \mathrm{kg})$ beginning at 7 days 
after inoculation. The same dosage of cilastatin (Wako Pure Chemical Industries, Ltd., Osaka, Japan), a DHP-I inhibitor, was also injected along with both agents. In the control group, saline was injected into mice instead of DRPM or MEPM. A total of 7 - 10 mice were used for each group.

\subsection{Bacteriological examination}

Mice were sacrificed by cervical dislocation on day 14 (12 h after final treatment). Lungs were dissected under aseptic conditions and were suspended in $1 \mathrm{ml}$ of saline. Organs were homogenized with a homogenizer (AS One Co., Osaka, Japan), were quantitatively inoculated onto Muller-Hinton II agar plates using serial dilutions, and were incubated at $37^{\circ} \mathrm{C}$ for $18 \mathrm{~h}$.

\subsection{Histological examination}

Mice were sacrificed by cervical dislocation on day 14 (12 h after final treatment). Lungs were fixed in $10 \%$ buffered formalin, and were stained with hematoxylin-eosin.

\subsection{Statistical analysis}

Bacterial data are expressed as means $\pm \mathrm{SD}$. Differences between groups were examined for statistical significance by unpaired U test. A $p$ value of less than 0.05 denoted the presence of a statistically significant difference. 


\section{Results}

\subsection{In vitro susceptibility}

Against P. aeruginosa S10, the MICs of DRPM and MEPM were 0.25 and $0.5 \mu \mathrm{g} / \mathrm{ml}$, respectively.

\subsection{Bacteriological examination (Fig. 1)}

The mean colony-forming units (CFU) $\pm \mathrm{SD}$ of $P$. aeruginosa recovered from homogenized lung tissue after treatment are shown in Figure 1. The mean number of viable bacteria in the lungs of DRPM, MEPM and control mice was $2.01 \pm 0.69,2.03 \pm 0.48$ and $3.90 \pm 1.40 \log _{10}$ CFU/lung, respectively. The number of viable bacteria in the lungs of mice treated with DRPM or MEPM was significantly less than that in lungs of controls ( $\mathrm{p}<0.05$ for each comparison). There were no significant differences in the number of viable bacteria in the lungs between the DRPM- and MEPM-treated mice $(\mathrm{p}=0.99)$.

\subsection{Histopathological examination (Fig. 2)}

The histopathological findings of lung specimens from mice sacrificed at $12 \mathrm{~h}$ after the final treatment are shown in Figure 2. In the control group, microscopic examination of the lung specimens confirmed the features of chronic bronchitis. Inflammatory cells had infiltrated around the 
bronchi and exudates had collected in the alveolar spaces. However, both the DRPM- and MEPM-treated groups showed fewer inflammatory cells and exudates than the control group. There were no significantly different findings between the DRPM- and MEPM-treated groups.

\section{Discussion}

DRPM was developed in Japan for use as a single agent in various infectious diseases.

In Europe and the United States, DRPM has been approved for the treatment of

hospital-acquired pneumonia, complicating urinary tract infections and intra-abdominal

infections, by the European Medicines Evaluation Agency and the US Food and Drug

Administration (US-FDA), respectively[9],[10]. DRPM has wide-spectrum antibacterial activity against gram-positive and gram-negative bacteria, and is particularly effective against $P$. aeruginosa in vitro when compared with other carbapenems, such as MEPM. P. aeruginosa is now being recognized as a relevant pathogen in chronic obstructive pulmonary disease (COPD) [11]. In COPD, after a clone is established, it remains in the lung for long periods, and can develop increasing degrees of antibiotic resistance and strong diversification [11]. 
Araki et al., Page 10 of 14

Carbapenems are hydrolyzed at the $\beta$-lactam ring by mammalian DHP-I $[12,13]$.

Therefore, IPM requires the DHP-I inhibitor cilastatin when used for therapy in humans.

However, 1- $\beta$-methylcarbapenems such as MEPM, ertapenem and DRPM show high stability in the presence of human DHP-I [14] and do not require a DHP-I inhibitor. On the other hand, DHP-I activity against DRPM and MEPM varies greatly with experimental animal species [15]. To minimize the effects of murine DHP-I, we treated mice with cilastatin in both the DRPM and MEPM treatment groups in this study.

In the present study, we investigated that the in vivo activities of DRPM and MEPM toward P. aeruginosa in a chronic respiratory infection mouse model. DRPM was found to reduce the number of viable bacteria and to prevent inflammation in the lungs to almost the same degree as MEPM. DRPM is as effective as MEPM in adults with complicating intra-abdominal infection, and its safety profile is not significantly different [16]. Furthermore, in patients with ventilator-associated pneumonia, 4-h intravenous infusion of DRPM was clinically efficacious and therapeutically noninferior to IPM [17]. These observations are consistent with results of our study.

We evaluated the antibacterial profile of DRPM against $P$. aeruginosa S10 strain in vivo, and the present results can be applied to clinical practice for chronic respiratory tract infections. 


\section{References}

1 Jones RN, Huynh HK, Biedenbach DJ, Fritsche TR \& Sader HS. Doripenem (S-4661), a novel carbapenem: comparative activity against contemporary pathogens including bactericidal action and preliminary in vitro methods evaluations. J Antimicrob Chemother. 2004;54:144-54. Fritsche TR, Stilwell MG \& Jones RN. Antimicrobial activity of doripenem (S-4661): a global surveillance report (2003). Clin Microbiol Infect. 2005;11:974-84. Brown SD \& Traczewski MM. Comparative in vitro antimicrobial activity of a new carbapenem, doripenem: tentative disc diffusion criteria and quality control. J Antimicrob Chemother. 2005;55:944-9. Wexler HM, Engel AE, Glass D \& Li C. In vitro activities of doripenem and comparator agents against 364 anaerobic clinical isolates. Antimicrob Agents Chemother. 2005;49:4413-7.

$5 \quad$ Ge Y, Wikler MA, Sahm DF, Blosser-Middleton RS \& Karlowsky JA. In vitro antimicrobial activity of doripenem, a new carbapenem. Antimicrob Agents Chemother. 2004;48:1384-96.

6 Jones RN, Sader HS \& Fritsche TR. Comparative activity of doripenem and three other carbapenems tested against Gram-negative bacilli with various beta-lactamase resistance mechanisms. Diagn Microbiol Infect Dis. 2005;52:71-4.

7 Yanagihara K, Tomono K, Sawai T, Hirakata Y, Kadota J, Koga H et al. Effect of clarithromycin on lymphocytes in chronic respiratory Pseudomonas aeruginosa infection. Am J Respir Crit Care Med. 1997;155:337-42.

8 Morinaga Y, Yanagihara K, Nakamura S, Yamamoto K, Izumikawa K, Seki M et al. In vivo efficacy and pharmacokinetics of tomopenem (CS-023), a novel carbapenem, against Pseudomonas aeruginosa in a murine chronic respiratory tract infection model. J Antimicrob Chemother. 2008;62:1326-31.

9 Van Wart SA, Andes DR, Ambrose PG \& Bhavnani SM. Pharmacokinetic-pharmacodynamic modeling to support doripenem dose regimen optimization for critically ill patients. Diagn Microbiol Infect Dis. 2009;63:409-14.

10 Livermore DM. Doripenem: antimicrobial profile and clinical potential. Diagn Microbiol Infect Dis. 2009;63:455-8.

11 Martinez-Solano L, Macia MD, Fajardo A, Oliver A \& Martinez JL. Chronic Pseudomonas aeruginosa infection in chronic obstructive pulmonary disease. Clin Infect Dis. 2008;47:1526-33.

12 Fukasawa M, Sumita Y, Harabe ET, Tanio T, Nouda H, Kohzuki T et al. Stability of 
meropenem and effect of 1 beta-methyl substitution on its stability in the presence of renal dehydropeptidase I. Antimicrob Agents Chemother. 1992;36:1577-9.

13 Kropp H, Sundelof JG, Hajdu R \& Kahan FM. Metabolism of thienamycin and related carbapenem antibiotics by the renal dipeptidase, dehydropeptidase. Antimicrob Agents Chemother. 1982;22:62-70.

14 Zhanel GG, Wiebe R, Dilay L, Thomson K, Rubinstein E, Hoban DJ et al. Comparative review of the carbapenems. Drugs. 2007;67:1027-52.

15 Tsuji M, Ishii Y, Ohno A, Miyazaki S \& Yamaguchi K. In vitro and in vivo antibacterial activities of S-4661, a new carbapenem. Antimicrob Agents Chemother. 1998;42:94-9.

16 Lucasti C, Jasovich A, Umeh O, Jiang J, Kaniga K \& Friedland I. Efficacy and tolerability of IV doripenem versus meropenem in adults with complicated intra-abdominal infection: a phase III, prospective, multicenter, randomized, double-blind, noninferiority study. Clin Ther. 2008;30:868-83.

17 Chastre J, Wunderink R, Prokocimer P, Lee M, Kaniga K \& Friedland I. Efficacy and safety of intravenous infusion of doripenem versus imipenem in ventilator-associated pneumonia: a multicenter, randomized study. Crit Care Med. 2008;36: 1089-96. 


\section{Legends to figures}

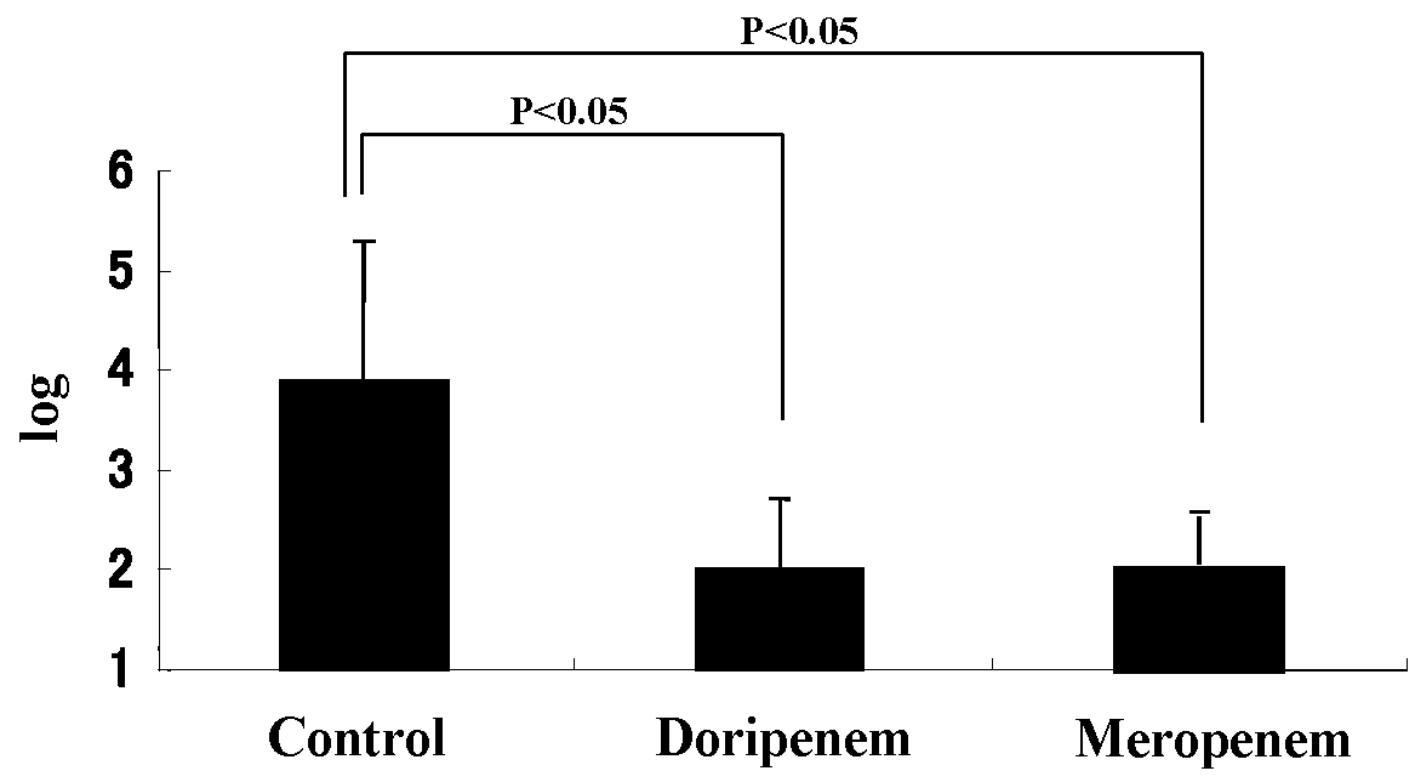

Fig. 1. Number of viable bacteria in the lungs of mice treated with doripenem or meropenem (100

$\mathrm{mg} / \mathrm{kg}$, twice daily) and in the lungs of control mice (saline, twice daily). Data are expressed as means \pm SD for $7-10$ mice. Doripenem and meropenem significantly reduced the number of viable bacteria when compared with controls $(\mathrm{p}<0.05)$. 


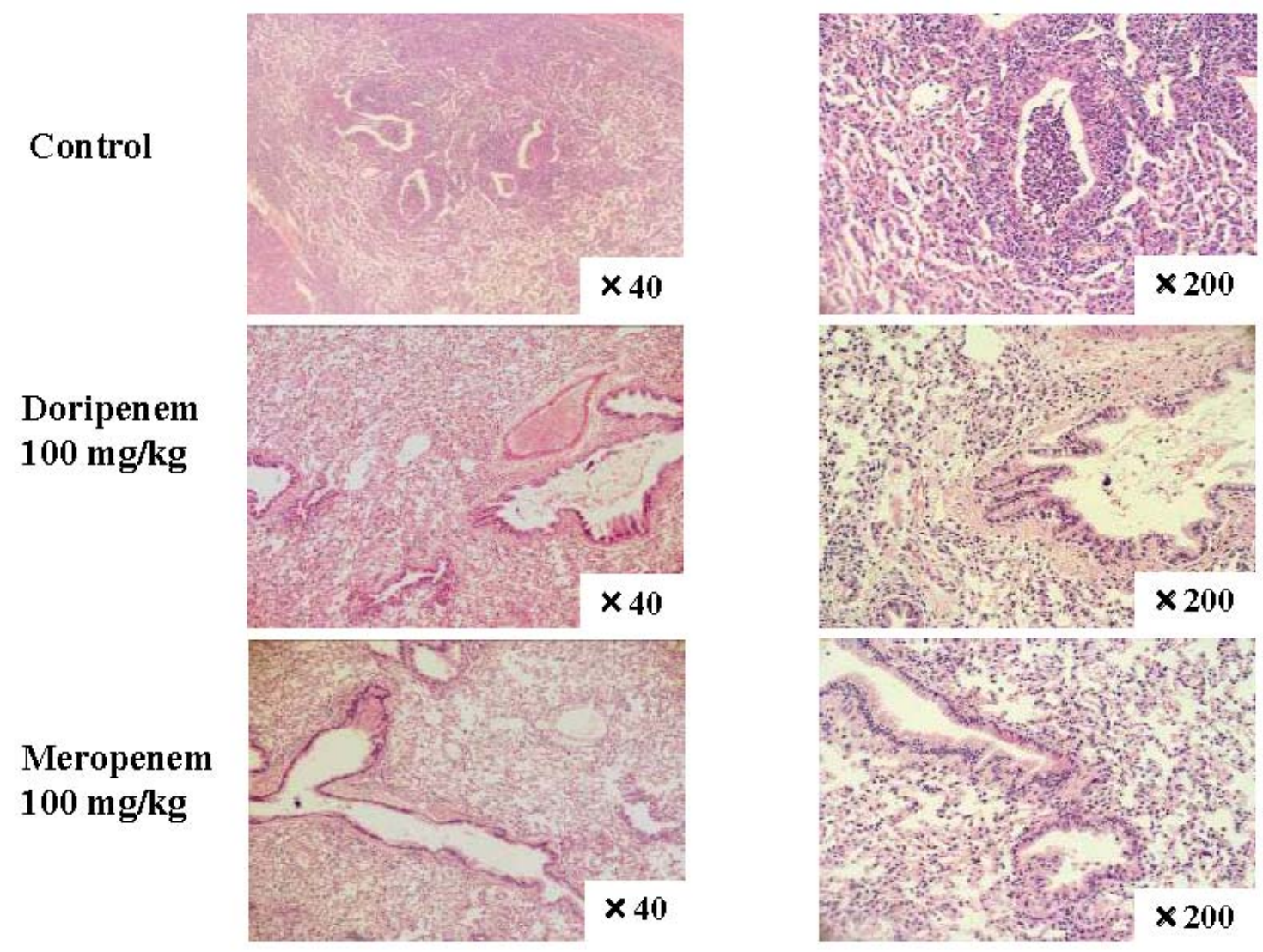

Fig. 2. Histopathological examination of lung specimens from mice sacrificed 14 days after infection with $P$. aeruginosa. The control group showed the features of chronic bronchopneumonia, with infiltration of inflammatory cells. However, few inflammatory cells were observed in the doripenemand meropenem-treated groups. 